\title{
Siewert type II adenocarcinoma of esophagogastric junction: treatment status
}

\author{
Shu-Chun Li, ${ }^{1,2}$, Lu Zang ${ }^{1,2}$ \\ 'Department of Surgery, Ruijin Hospital, Shanghai Jiao Tong University School of Medicine, Shanghai 200025, China. \\ ${ }^{2}$ Shanghai Minimally Invasive Surgery Center, Shanghai 200025, China.
}

Correspondence to: Dr. Lu Zang, Department of Surgery, Ruijin Hospital, Shanghai Jiao Tong University School of Medicine, 197 Ruijin Er Road, Shanghai 200025, China. Shanghai Minimally Invasive Surgery Center, Shanghai 200025, China. E-mail: zanglu@yeah.net

How to cite this article: Li SC, Zang L. Siewert type II adenocarcinoma of esophagogastric junction: treatment status. Miniinvasive Surg 2019;3:15. http://dx.doi.org/10.20517/2574-1225.2018.13

Received: 31 Dec 2018 First Decision: 25 Mar 2019 Revised: 8 Apr 2019 Accepted: 17 Apr 2019 Published: 15 May 2019

Science Editor: Tetsu Fukunaga Copy Editor: Cai-Hong Wang Production Editor: Huan-Liang Wu

\begin{abstract}
The incidence of adenocarcinoma of esophagogastric junction (AEG) has been increased continuously in the past decades, especially in western countries. Siewert type II is regard as the true AEG because of its location, however, the treatment for Siewert type II AEG has not reached a consensus in the academic. According to published studies nowadays, this commentary will introduce the surgical strategies and put forward suggestions for Siewert type II AEG in several aspects as follows: (1) optimal surgical approach; (2) optimal extent of lymph node dissection; (3) reconstruction methods. With the development of minimally invasive surgery, many experienced surgeons perform esophagogastrostomy via transhiatal approach. Moreover, many details during the surgery still need further research by cooperation between different departments and even countries.
\end{abstract}

Keywords: Esophagogastric junction, adenocarcinoma, surgical approach, reconstruction

\section{INTRODUCTION}

The incidence of adenocarcinoma of esophagogastric junction (AEG) has been increased continuously in the past decades, especially in western countries ${ }^{[1,2]}$. AEG refer to the adenocarcinoma which straddle the gastroesophageal junction (EGJ). EGJ is the region where the esophagus joins the $\operatorname{stomach}^{[3]}$. There are different methods to identify the EGJ including surgical, physiology, histology, endoscopy or upper gastrointestinal imaging ${ }^{[4,5]}$. In terms of conveniences, the surgeons usually regard the boundary of the tubular esophagus and the saccular stomach as EGJ in the surgery.

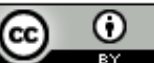

(C) The Author(s) 2019. Open Access This article is licensed under a Creative Commons Attribution 4.0 International License (https://creativecommons.org/licenses/by/4.0/), which permits unrestricted use, sharing, adaptation, distribution and reproduction in any medium or format, for any purpose, even commercially, as long as you give appropriate credit to the original author(s) and the source, provide a link to the Creative Commons license, and indicate if changes were made. 
Siewert classification, one of the most widely used classifications today for AEG, was come up by Siewert in $1987^{[6]}$. In this classification, AEG was defined as the tumor whose epicenter located within $5 \mathrm{~cm}$ oral and aboral of the esophagogastric junction ${ }^{[7]}$. Siewert type I refers to the tumor whose epicenter located $1-5 \mathrm{~cm}$ above the EGJ, and it is usually regarded as esophagus cancer. Siewert type II tumor is considered as true AEG with a center within $1 \mathrm{~cm}$ above and $2 \mathrm{~cm}$ below EGJ. Siewert type III, with whose center located $2-5 \mathrm{~cm}$ below the EGJ, is treated as gastric cardia tumors. Because AEG has unusual oncology behavior and characteristics, the treatment for type II remains controversial. The aim of this commentary is to introduce the surgical strategies for type II AEG in recent years.

\section{OPTIMAL SURGICAL APPROACH FOR SIEWERT TYPE II AEG}

For advanced AEG, surgery remains predominant treatment because of the possibility of lymph node involvement. The appropriate surgical approach should take a lot into consideration including oncology safety, lymph node dissection and resection margin involvement. In Japan, no matter which type of AEG is treated by upper gastrointestinal surgeons. However, in China, gastric cancer belongs to abdominal surgery, and esophagus cancer belongs to thoracic surgery. For Siewert type II AEG, transthoracic and transhiatal are two major surgical approach. Different surgeons have different opinions on surgical approach.

The Japan Clinical Oncology Group (JCOG) conducted a randomized controlled multicenter clinical trial in Japan (JCOG9502). 167 patients with cancer of Siewert type II or III were enrolled from 27 hospitals in japan and randomly assigned to abdominal transhiatal approach (TH) or left thoracoabdominal (LTA). The aim of this trial was to explore whether LTA, compared with TH, could prolong the overall survival. The 5 -year overall survival was $52.3 \%$ and $37.9 \%$ in the TH group and LTA group, respectively, but the morbidity was worse in LTA than $\mathrm{TH}^{[8]}$. Subgroup analyses showed no survival benefit for Siewert II patients in LTA group. Moreover, with respect of six selected major complications (pancreatic fistula, abdominal abscess, pneumonia, anastomotic leak, empyema thoracis and mediastinitis), the incidence was significantly higher following the LTA than the TH group: $41 \%$ vs. $22 \%(P=0.008)$. And there were two treatment-related death in LTA group ${ }^{[9]}$. Therefore, LTA approach is not recommended for Siewert type II AEG with the length of esophagus invasion $\leq 3 \mathrm{~cm}$.

Another trial focused on the best approach for esophageal carcinoma. It randomly assigned 220 patients with adenocarcinoma of mid-to-distant esophagus or adenocarcinoma of the gastric cardia involving the distal esophagus to transhiatal esophagectomy (THE) or right transthoracic esophagectomy (TTE, Ivor-Lewis) with extended lymphadenectomy. Ivor-Lewis is a right transthoracic surgical approach for distal esophagus cancer, which was introduced by Lewis in $1946^{[10]}$. And this approach was widely used in western countries. This approach has two procedure which includes gastrectomy and abdominal lymphadenectomy for stage I, then a right thoracotomy with esophageal resection and peri-esophageal lymphadenectomy for stage II. Ivor-Lewis was associated with more in-hospital morbidity than transhital. After transhiatal and transthoracic resection, 5-year survival was 34\% and 36\% $(P=0.71)$, respectively. Although no statistically difference founded among groups, there was a trend toward improving longterm survival at five years with Ivor-Lewis approach. In subgroup analysis based on Siewert classification, a 5-year survival benefit of $14 \%$ was found in transthoracic group for Siewert type I ( $51 \% v s .37 \%, P=0.33$ ) than transhital group ${ }^{[11,12]}$, which indicated that Ivor-Lewis may have no benefits for type II AEG. It may be related to TTE could gain more involved lymph nodes than THE in type I AEG. Recently a single center reviewed 242 Siewert type II AEG retrospectively, of whom 56 (23.1\%) underwent thoracoabdominal esophagectomy (TAE) and $186(76.9 \%)$ had a transhiatal extended gastrectomy (THG). No difference in morbidity $(P=0.197)$ and mortality $(P=0.711)$ were observed, including anastomotic leakages $(P=0.625)$ and pulmonary complications $(P=0.494)$. And the number of resected lymph node and rate of Ro resection 
have no difference as well. Overall survival after TAE was significantly longer than after THG (33.6 months vs. not reached, $P=0.02)^{[13]}$. It suggested TAE isn't worse than THG for Siewert type II AEG, especially for advanced patients who had neoadjuvant chemotherapy. But this study didn't report the length of esophagus invasion, which may influence the surgical approach chosen.

In terms of surgical extent of type II AEG, it is related to surgical approach. In order to evaluate the worldwide trends in surgical techniques for esophageal cancer surgery, a worldwide survey was performed among surgeons. In Asia gastrectomy was more popular, whereas in North America the majority procedure was esophagectomy ${ }^{[14]}$. And thoracic surgeons may prefer distal esophagectomy, while abdominal surgeons prefer proximal or total gastrectomy via transhital approach. And a sufficient resection margin is another prognostic factor for oncology safety. Mine et al ${ }^{[15]}$ demonstrated that proximal margin length of more than $20 \mathrm{~mm}$ in resected specimens seem satisfactory for patients with type II AEG by transhital approach. Frozen section examination of the resection line is recommended by the Japanese gastric cancer treatment guidelines to ensure an Ro resection ${ }^{[16]}$.

On the basis of the best evidence so far, JCOG 9502, for Siewert type II AEG with esophagus invasion of 3 $\mathrm{cm}$ or less, transhiatal approach is safety and effective. It is necessary to conduct well-designed multicenter clinical trials to investigate appropriate approach for type II AEG with a larger lesion.

\section{OPTIMAL EXTENT OF LYMPH NODE DISSECTION FOR SIEWERT TYPE II AEG}

Siewert type II AEG is located in the boundary of distal esophagus and gastric cardia, the pathway of lymph metastasis is not same as esophagus or gastric cancer alone. In previous retrospective study, mediastinal lymph node involvement rate was $46.2 \%-65.0 \%$ for type I, $12.0 \%-29.5 \%$ for type II, and $6.0 \%-9.3 \%$ for type III tumors ${ }^{[17,18]}$. Nunobe et al. ${ }^{[19]}$ claimed that the more esophagus invaded, the higher lymph node metastasis rate is.

The optimal extent of lymph node dissection for Siewert type II AEG remain uncertain. A nation-wide retrospective study of lymphadenectomy for EGJ cancer was conducted in 2012 in Japan ${ }^{[20]} .2807$ patients without preoperative therapy were included in the analysis. The frequency of dissection for mediastinal lymph node was higher in esophagus-predominant cancer than stomach-predominant cancer. With respect to esophagus-predominant cancer, the lymph node dissection rate is higher in lower mediastinal lymph node than upper or middle mediastinal (40\%vs. 15\%). For stomach-predominant cancer, the mediastinal lymph node dissection focuses mainly on lower mediastinal, and advanced cancer especially. The possibolity of metastasis rose as the $\mathrm{pT}$ stage increased, and rates of metastasis is high in No. 1, 2, 3, 7 . Mediastinal lymph node metastasis could be found in esophagus-predominant cancer, especially in lower mediastinal. On the contrast, it is rare in stomach-predominant. And rates of metastasis at No. 4sa, 4sb, 4d, 5 and 6 were very low, despite their high dissection rates especially in stomach-predominant cancer cases. Therefore, lymph node metastasis is mainly in the abdominal and lower mediastinal for Siewert type II AEG. Another study has similar result. It reviewed 381 Siewert type II AEG retrospectively. The nodal metastasis mainly founded in No. 1, 2, 3, 7, 11p and lower mediastinal. The middle and upper mediastinal metastasis rate is low, but related to extremely poor prognosis ${ }^{[21]}$.

It still doesn't have a standard lymph node dissection extent. The Japanese Gastric Cancer Association published a temporary lymphadenectomy guideline for junctional cancer $\leq 4 \mathrm{~cm}$. It is based on the tumor location, histology and T-categories ${ }^{[16]}$. Abdominal lymph node dissection can refer to gastric cancer, and No 4, 5, 6 can be omitted in early stage cases, because of low metastasis rate. Lower mediastinal should dissect routinely, but upper and middle mediastinal not. According to personal experience, lower thoracic paraesophageal lymph nodes (No. 110) and supradiaphragmatic lymph nodes (No. 111) can be resected via transhital approach, but it's difficult to resect posterior mediastinal lymph nodes (No. 112) because of 
limited space. In terms of peri-esophageal lymphadenectomy, transthoracic approach may have superiority for en bloc lymphadenectomy ${ }^{[22]}$.

\section{RECONSTRUCTION METHODS FOR SIEWERT TYPE II AEG}

Reconstruction is one of the most difficult steps during the surgery. For early type II AEG, lower esophagectomy plus proximal gastrectomy seems enough, because of low para-stomach nodal metastasis rate in No. 4, 5 and 6. While as for advanced type II AEG, lower esophagectomy plus total gastrectomy is essential ${ }^{[23]}$. There are many reconstruction methods for proximal gastrectomy, including esophagogastrostomy, gastric tube reconstruction ${ }^{[24,25]}$, double tract ${ }^{[26]}$, different kinds of jejunal interposition and double flap method ${ }^{[27,28]}$. The major concern for proximal gastrectomy is the high incidence of postoperative complications, reflux esophagitis especially, which is a negative factor for quality of life. Many reconstruction methods are meant to resist reflux, while it remains lacking consensus. A retrospective study demonstrated that double tract reconstruction is a simple and effective method in decreasing reflux esophagitis compared with Roux-en- $\mathrm{Y}^{[29]}$. It still needs RCT to provide high-grade evidence for the method.

Compared with open surgery, minimally invasive surgery has unique advantages, such as acceptable lymph node retrieval, good postoperative outcomes, and low mortality ${ }^{[30]}$. Many experienced surgeons choose minimally invasive surgery for type II $\mathrm{AEG}^{[31]}$. Laparoscopic-assisted proximal gastrectomy is prevalent for type II AEG. Because circular stapler can finish a higher anastomosis level, it is easier for type II AEG with Ro resection. And the key procedure of using circular stapler in totally laparoscopic procedure is placing the anvil to the stump of esophagus. Transorally inserted anvil (OrVil) ${ }^{[31,32]}$ and hemidouble stapling technique $^{[33]}$ are two easy methods to accomplish that. Recently, it has become more and more popular for totally laparoscopic technique. Liner stapler is a good choice for totally laparoscopic anastomosis, such as Overlap method ${ }^{[34]}$. But compared with laparoscopic-assisted gastrectomy, which could provide some reference for the border of tumor through a sense of touch, it is harder to ensure Ro resection, especially in advanced type II AEG. In addition, there is little space to use such devices, especially for type II AEG which needs a high anastomosis level. Takiguchi et al.$^{[35]}$ introduce laparoscopic mediastinal dissection via an open left diaphragm approach for advanced type II AEG. Firstly, divide phrenicoesophageal ligament around the esophagus along the esophageal hiatus. Then, open the left side of mediastinal pleura and incise the left diaphragm with a $60-\mathrm{mm}$ linear stapler. So that, it creates a clear space and a good view for the further mediastinal lymph node dissection and reconstruction. Although no severe complication was seen in this study, it is still unknown whether this method is more harmful compared with transthoracic approach like Ivor-Lewis. The method may lead to a larger trauma which needs to place an intrathoracic drainage tube. And a larger sample size was needed to provide more evidence.

The incidence rate of AEG has been on the rise for decades. Siewert classification has become the standard classification for AEG. Siewert type I AEG should be treated as esophagus cancer, while type III should be regarded as gastric cancer. Because of the unique location of Siewert type II AEG, the treatment still doesn't reach consensus. Surgery remains the fundamental treatment, a lot of detail during surgery are needed to research in the future.

\section{DECLARATIONS}

\section{Authors' contributions}

Concept and design: Zang L

Manuscript preparation: Li SC

Manuscript review: Zang L

\section{Availability of data and materials}

Not applicable. 


\section{Financial support and sponsorship}

None.

\section{Conflicts of interest}

Both authors declared that there are no conflicts of interest.

\section{Ethical approval and consent to participate}

Not applicable.

\section{Consent for publication}

Not applicable.

\section{Copyright}

(C) The Author(s) 2019.

\section{REFERENCES}

1. Dubecz A, Solymosi N, Stadlhuber RJ, Schweigert M, Stein HJ, et al. Does the incidence of adenocarcinoma of the esophagus and gastric cardia continue to rise in the twenty-first century?-a SEER database analysis. J Gastrointest Surg 2013.

2. Zhou Y, Zhang Z, Zhang Z, Wu J, Ren D, et al. A rising trend of gastric cardia cancer in Gansu Province of China. Cancer Lett 2008;269:18-25.

3. Oezcelik A, DeMeester SR. General anatomy of the esophagus. Thorac Surg Clin 2011;21:289-97.

4. Odze RD. Pathology of the gastroesophageal junction. Semin Diagn Pathol 2005;22:256-65.

5. Wallner B. Endoscopically defined gastroesophageal junction coincides with the anatomical gastroesophageal junction. Surg Endosc 2009;23:2155-8.

6. Siewert JR, Holscher AH, Becker K, Gossner W. Cardia cancer: attempt at a therapeutically relevant classification. Chirurg 1987;58:2532.

7. Siewert JR, Stein HJ. Carcinoma of the gastroesophageal junction - classification, pathology and extent of resection. Dis Esophagus 1996;9:173-82.

8. Sasako M, Sano T, Yamamoto S, Sairenji M, Arai K, et al. Left thoracoabdominal approach versus abdominal-transhiatal approach for gastric cancer of the cardia or subcardia: a randomised controlled trial. Lancet Oncol 2006;7:644-51.

9. Kurokawa Y, Sasako M, Sano T, Yoshikawa T, Iwasaki Y, et al. Ten-year follow-up results of a randomized clinical trial comparing left thoracoabdominal and abdominal transhiatal approaches to total gastrectomy for adenocarcinoma of the oesophagogastric junction or gastric cardia. Br J Surg 2015;102:341-8.

10. Lewis I. The surgical treatment of carcinoma of the oesophagus; with special reference to a new operation for growths of the middle third. Br J Surg 1946;34:18-31.

11. Omloo JM, Lagarde SM, Hulscher JB, Reitsma JB, Fockens P, et al. Extended transthoracic resection compared with limited transhiatal resection for adenocarcinoma of the mid/distal esophagus: five-year survival of a randomized clinical trial. Ann Surg 2007;246:9921000; discussion 1000-1.

12. Hulscher JB, van Sandick JW, de Boer AG, Wijnhoven BP, Tijssen JG, et al. Extended transthoracic resection compared with limited transhiatal resection for adenocarcinoma of the esophagus. N Engl J Med 2002;347:1662-9.

13. Blank S, Schmidt T, Heger P, Strowitzki MJ, Sisic L, et al. Surgical strategies in true adenocarcinoma of the esophagogastric junction (AEG II): thoracoabdominal or abdominal approach? Gastric Cancer 2018;21:303-14.

14. Haverkamp L, Seesing MF, Ruurda JP, Boone J, R VH. Worldwide trends in surgical techniques in the treatment of esophageal and gastroesophageal junction cancer. Dis Esophagus 2017;30:1-7.

15. Mine S, Sano T, Hiki N, Yamada K, Kosuga T, et al. Proximal margin length with transhiatal gastrectomy for Siewert type II and III adenocarcinomas of the oesophagogastric junction. Br J Surg 2013;100:1050-4.

16. Japanese Gastric Cancer Association. Japanese gastric cancer treatment guidelines 2014 (ver. 4). Gastric Cancer 2017;20:1-19.

17. Pedrazzani C, de Manzoni G, Marrelli D, Giacopuzzi S, Corso G, et al. Lymph node involvement in advanced gastroesophageal junction adenocarcinoma. J Thorac Cardiovasc Surg 2007;134:378-85.

18. Siewert JR, Stein HJ, Feith M. Adenocarcinoma of the esophago-gastric junction. Scand J Surg 2006;95:260-9.

19. Nunobe S, Ohyama S, Sonoo H, Hiki N, Fukunaga T, et al. Benefit of mediastinal and para-aortic lymph-node dissection for advanced gastric cancer with esophageal invasion. J Surg Oncol 2008;97:392-5.

20. Yamashita H, Seto Y, Sano T, Makuuchi H, Ando N, et al. Results of a nation-wide retrospective study of lymphadenectomy for esophagogastric junction carcinoma. Gastric Cancer 2017;20:69-83.

21. Yoshikawa T, Takeuchi H, Hasegawa S, Nozaki I, Kishi K, et al. Theoretical therapeutic impact of lymph node dissection on adenocarcinoma and squamous cell carcinoma of the esophagogastric junction. Gastric Cancer 2016;19:143-9.

22. Barreto JC, Posner MC. Transhiatal versus transthoracic esophagectomy for esophageal cancer. World J Gastroenterol 2010;16:3804-10.

23. Mariette C, Piessen G, Briez N, Gronnier C, Triboulet JP. Oesophagogastric junction adenocarcinoma: which therapeutic approach? Lancet Oncol 2011;12:296-305. 
24. Shiraishi N, Hirose R, Morimoto A, Kawano K, Adachi Y, et al. Gastric tube reconstruction prevented esophageal reflux after proximal gastrectomy. Gastric Cancer 1998;1:78-9.

25. Chen XF, Zhang B, Chen ZX, Hu JK, Dai B, et al. Gastric tube reconstruction reduces postoperative gastroesophageal reflux in adenocarcinoma of esophagogastric junction. Dig Dis Sci 2012;57:738-45.

26. Aikou T, Natsugoe S, Shimazu H, Nishi M. Antrum preserving double tract method for reconstruction following proximal gastrectomy. Jpn J Surg 1988;18:114-5.

27. Mine S, Nunobe S, Watanabe M. A novel technique of anti-reflux esophagogastrostomy following left thoracoabdominal esophagectomy for carcinoma of the esophagogastric junction. World J Surg 2015;39:2359-61.

28. Muraoka A, Kobayashi M, Kokudo Y. Laparoscopy-assisted proximal gastrectomy with the hinged double flap method. World J Surg 2016;40:2419-24.

29. Xiao JW, Liu ZL, Ye PC, Luo YJ, Fu ZM, et al. Clinical comparison of antrum-preserving double tract reconstruction vs roux-en-Y reconstruction after gastrectomy for Siewert types II and III adenocarcinoma of the esophagogastric junction. World J Gastroenterol 2015;21:9999-10007.

30. Wiesel O, Whang B, Cohen D, Fisichella PM. Minimally invasive esophagectomy for adenocarcinomas of the gastroesophageal junction and distal esophagus: notes on technique. J Laparoendosc Adv Surg Tech A 2017;27:162-9.

31. Kinoshita T, Gotohda N, Kato Y, Takahashi S, Konishi M, et al. Laparoscopic transhiatal resection for Siewert type II adenocarcinoma of the esophagogastric junction: operative technique and initial results. Surg Laparosc Endosc Percutan Tech 2012;22:e199-203.

32. Jeong O, Park YK. Intracorporeal circular stapling esophagojejunostomy using the transorally inserted anvil (OrVil) after laparoscopic total gastrectomy. Surg Endosc 2009;23:2624-30.

33. Omori T, Oyama T, Mizutani S, Tori M, Nakajima K, et al. A simple and safe technique for esophagojejunostomy using the hemidouble stapling technique in laparoscopy-assisted total gastrectomy. Am J Surg 2009;197:e13-7.

34. Inaba K, Satoh S, Ishida Y, Taniguchi K, Isogaki J, et al. Overlap method: novel intracorporeal esophagojejunostomy after laparoscopic total gastrectomy. J Am Coll Surg 2010;211:e25-9.

35. Takiguchi S, Miyazaki Y, Shinno N, Makino T, Takahashi T, et al. Laparoscopic mediastinal dissection via an open left diaphragm approach for advanced Siewert type II adenocarcinoma. Surg Today 2016;46:129-34. 